\title{
CONDENSATION NUCLEI OBTAINED FROM THE EVAPORATION OF FOG PARTICLES. ${ }^{1}$
}

\author{
BY C. BARUS.
}

I. Standardization with Ions.-A curious behavior appeared in an attempt to standardize the coronas of cloudy condensation by aid of the ions due to gamma rays penetrating the fog chamber. These were obtained from a sample of radium of strength $10,000 \times$ and weighing $100 \mathrm{mg}$., sealed in an aluminum tube. The coronas were produced by successive exhaustions of the same value and the fogs were dissipated by compression, filtered air being introduced as soon as possible. The data obtained in the experiments showed an enormous initial loss. To obtain large coronas, the exhaustion to catch the ions was necessarily higher (drop of pressure $\delta p=22.6$ ) than to catch the water nuclei resulting from the evaporation of fog particles $(\delta p=\mathrm{I} 7 . \mathrm{I})$; but this was allowed for.

The attempt to find the subsidence constant, $S$, fails ; as for instance, if $s / 30$ is the angular diameter of the coronas,

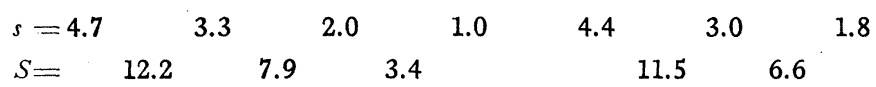

showing a well marked progression of data. Similarly the attempt to find the initial nucleation $n_{0}$ fails, as a progression is here equally manifest. In other words, with the evaporation of the first fog (precipitated on ions) more than one half the nuclei are lost, whereas in subsequent evaporations, the behavior of the remaining nuclei is more like phosphorus nuclei, without evaporation losses.

That the discrepancy is in large measure due to the losses in the first evaporation will be seen if the second residue

$$
\left(n \times \mathrm{IO}^{-3}=50.6\right)
$$

be taken as the initial number. The results are in thousands per cub. $\mathrm{cm}$.

${ }^{1}$ Condensed from a report (now in preparation) to the Carnegie Institution of Washington. 


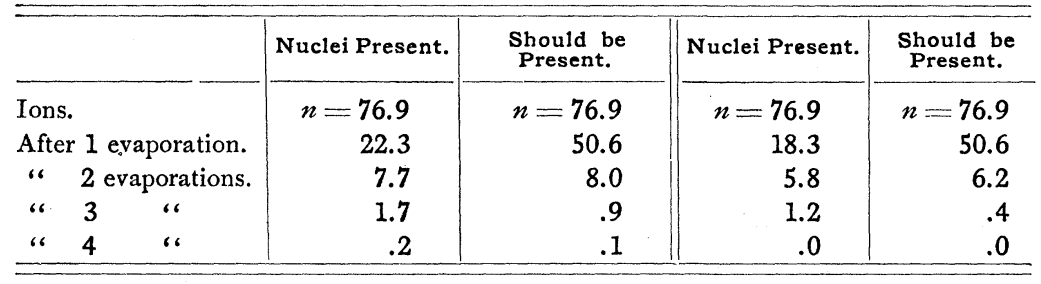

2. Systematic Data. - Thus it appears that the water nuclei obtained by evaporating fog particles precipitated on ions vanish more rapidly, at least in the beginning, than may be accounted for as the combined result of the exhaustion applied and the subsidence. New results were therefore investigated by aid of the diffraction method ${ }^{1}$ of two sources.

These are given in Figs. 1, 2, 3 and 4, where the ordinates show the number of nuclei caught in the successive identical exhaustions, and the abscissas the distributive number of the exhaustion

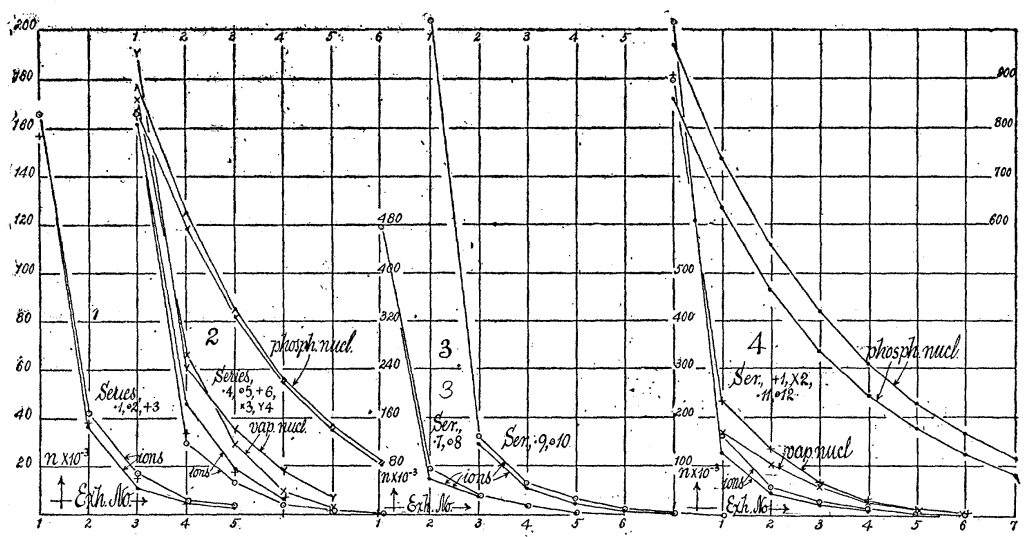

Figs. 1-4.

applied. Each exhaustion is followed by an evaporation of fog particles, whereby the residual water nuclei are produced for the next exhaustion in order.

In the first, second, and third series of experiments the exhaustion was somewhat above the condensation limit of air, so that the coronas do not vanish (Fig. I). But as the vapor nuclei are rela-

\footnotetext{
${ }^{1}$ See Am. Journal of Science, XXIV., pp. 277-28I, 1907.
} 
tively few as compared with the ions, the initial fall of nucleation is well brought out. The exhaustion is here identical for ions and for water nuclei.

In series $4,5,6$, the exhaustion for water nuclei is below the fog limit of air and the coronas vanish on successive exhaustion. It is necessary, therefore, to make the exhaustion for ions (only) above the condensation limit of air as otherwise too few would be caught. The observed march of data is however similar to the preceding experiments, as is shown in Fig. 2.

These results were now varied by bringing to bear stronger radiation obtained from an X-ray bulb placed at successively decreasing distances $D$ from the fog chamber. In series 7 and $8, D=40$; in series 9 and IO, $D=20 \mathrm{~cm}$; and in series II and I $2, D=\mathrm{I} 2 \mathrm{~cm}$. (about) from the axis of the fog chamber. The large initial radiations drop off rapidly in the same way as in the preceding case. All the series are consistent, except the eleventh, in which the initial drop is too large compared with the others. It was customary to keep the exhaust cock open for five seconds, after which the filter cock was opened to dispel the fog, one minute being allowed between the exhaustions. The results are shown in detail in the figures together with similar data for the vapor nuclei of dust-free air and for phosphorus nuclei.

3. Data for Vapor Nuclei. - The figures contain similar data for the vapor nuclei of wet dust-free air. In series I and 2 large coronas or high nucleations are met with at the start, and they are compared in Fig. 4 with a corresponding case for ions. In series 3 and 4 lower initial nucleations are contained and these data are compared in Fig. 2 with the corresponding cases of ions and phosphorus nuclei. Corrections for subsidence should have been added to the graphs for ions and for vapor nuclei, but these are not large enough to modify them materially, so far as the figures go.

4. Remarks on the Results. - The graphs in Figs. 2 and 4 show unmistakably that the water nuclei obtained from the evaporation of fog particles precipitated on ions, vanish in the successive exhaustion faster than in the corresponding case with the vapor nuclei of dust-free air; while the water nuclei from fog particles precipitated on vapor nuclei vanish much faster than is the case for the corre- 
sponding solutional nuclei obtained with phosphorus emanation. It is thus necessary to examine in detail the three more obvious causes for the decrease in the number of nuclei, which are as follows: (I) the exhaustions applied alike in all cases ; $(2)$ the subsidence of fog particles during the short time of their suspension, i. e., between the exhaustion and the evaporation by influx of air; (3) the occurrence of electrical charge in the case of ionized nuclei whereby the charged water nuclei may be brought to coalescence.

Probably the best method of reaching a numerical result will consist in eliminating the effect of exhaustion and subsidence as was done for phosphorus nuclei, thus leaving the new losses of nuclei alone outstanding. If $n_{z}^{\prime}$ is the nucleation of the $z$ th exhaustion,

$$
n_{z}^{\prime}=n_{1} y^{z-1} \Pi\left(I-S / s_{z-1}^{2}\right) \text {, }
$$

where $y$ is the exhaustion ratio and the product $\Pi\left(\mathrm{I}-S / s_{z-1}^{2}\right)$, the correction for subsidence, the data obtained are such as apply for solutional nuclei produced by phosphorus; but they are throughout enormously in excess of the values $n_{z}$ observed for vapor nuclei and for ions. Supposing that there is a second cause of dissipation with each exhaustion the equation may be written (abbreviating the products $\Pi$ )

$$
n_{z}^{\prime}=n_{1} y^{z-1} x^{z-1} I I
$$

merely to get a numerical statement of the case. The values of $x$ so found show a gradual increase, as the numbers of exhaustions increase or the nucleations. decrease and the sizes of fog particles increase, so that the greatest dissipation of nuclei occurs during the first exhaustion.

If these values of $x$ are summarized and constructed in terms of $n$, they also show that $x$ is considerably in excess for vapor nuclei as compared with ions.

The best method of interpretating the above results is in terms of an equation of the form (if $n_{1}$ be the initial nucleation)

$$
n_{z}=n_{1} y^{z-1} x x^{\prime} x^{\prime \prime} \ldots I \text {, }
$$

where $n_{z}$ is the nucleation of the zth exhaustion, $y$ the exhaustion ratio, the product $I I$ the subsidence correction and $x, x^{\prime}, x^{\prime \prime}$, etc., the successive coefficients showing the relative survival, $x$, or the 
corresponding loss, $(\mathrm{I}-x)$, of nuclei, accompanying each evapoartion of the fog particles. This equation asserts that the loss is different in the successive evaporations, which is actually the case in question.

So treated the results indicate the degree to which the first evaporation is preponderating and prove it to be more destructive, as the original number of ions is greater. Thus when $n=\mathrm{I} 60,000,(\mathrm{I}-x)$ or 60 to 70 per cent. are lost during the first and only about $\left(1-x^{\prime}\right)$ or 20 per cent. during the second or subsequent evaporations. If $n=900,000$ to $\mathrm{I}, \mathrm{I} 100,000$ (where the fog particles are very much smaller), the first destroys about 80 per cent., the second 40 per cent., the third 30 per cent. of the number which happen to be present just before the respective evaporation. Hence for large values of $n$ the loss due to evaporation is appreciable throughout many repetitions.

The results, for fog articles precipitated upon the vapor nuclei of dust-free air are similar, but in no case does the coefficient of survival $x$ increase after the first exhaustion, as was the case with ions. Contrasting the case of ions with the case for vapor nuclei, specifically, the coefficient of survival $x$ is always decidedly smaller for ions in the first exhaustion than for vapor nuclei. The charged nuclei are therefore destroyed in greater number than uncharged nuclei by the evaporation of fog particles precipitated on them. When the number of nuclei is large $\left(10^{6}\right)$, this is also true in subsequent evaporations, though the contrast is less marked.

Another question which comes up for settlement is this: Whether the fog particles which are represented by nuclei after evaporation are above a certain critical size, and those particles which vanish are below it. This is hardly probable because all the fog particles contribute to the same corona and because it implies an enormous inequality in the fog particles of the first exhaustion, seeing that 45 per cent. to 85 per cent. vanish in the different cases cited. These results for the diameters $d$ of fog particles and the corresponding coefficients of survival $x, x^{\prime}$, etc., are constructed graphically in Fig. 5, $a, b$, for ions and in Fig. 5, $c, d$, for water nuclei.

Fig. 5, $a$, containing series 4 to 8 for small ionized nucleations (below $n=500$ 000), suggests that $x$ may change abruptly when 
$d=.0006 \mathrm{~cm}$. ; while Fig. $5 b$, for large ionized nucleations, $10^{6}$, has the same appearance at $d=.0005 \mathrm{~cm}$. However, this is nothing more than the transition from the first to the second evaporation, the former being so much more efficient.

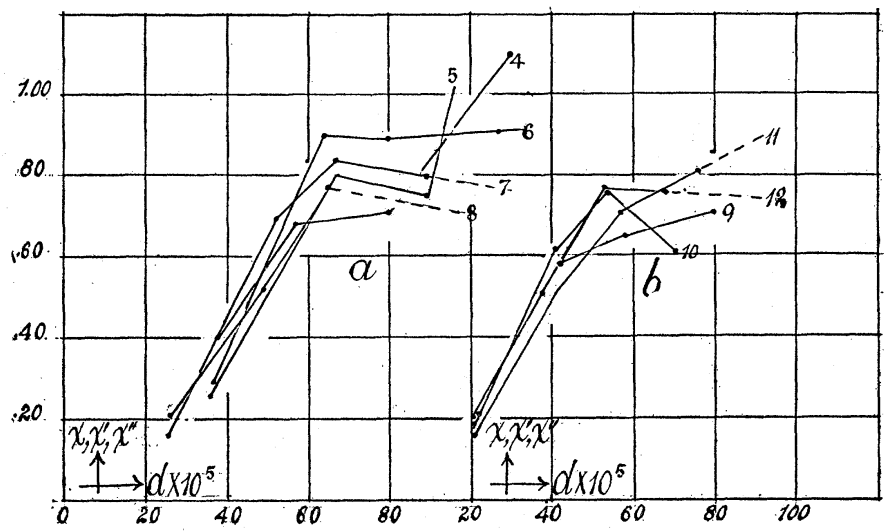

Jrig. 5.

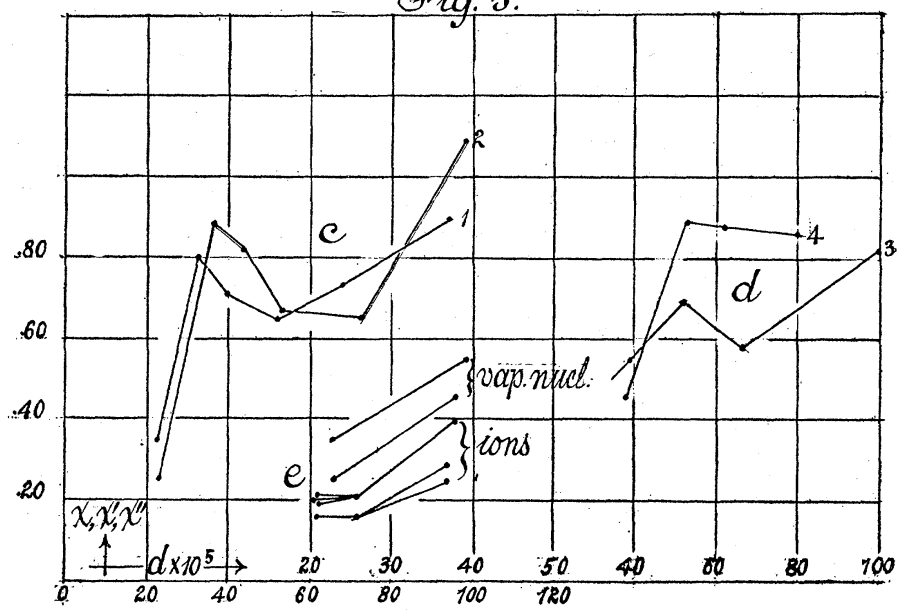

Fig. $5, c$ and $d$, for large and small nucleations of vapor nuclei has the same character. In $c$ for instance there is an abrupt change below 40,000 nuclei. But the case is again one instancing the paramount importance of the first evaporation.

There is, however, no doubt of an outstanding effect due to the number or the size of the nuclei. The coefficient of survival $x$ de- 
creases as the number of nuclei increases, or better as their size diminishes. Thus if the comparison be restricted to the first evaporation, Fig. $5 e$,

$$
\begin{aligned}
& \text { Ions: }\left\{\begin{array}{lllllllll}
10^{5} d=38 & 37 & 37 & 26 & 26 & 21 & 22 & 21 & 21 \mathrm{~cm} \\
10^{2} & \text {. }
\end{array}\right. \\
& \text { Vapor nuclei : }\left\{\begin{array}{lll}
10^{5} d=39 & 38 & 23 \\
10^{2} x & 23 \mathrm{~cm}
\end{array}\right. \text {. } \\
& \begin{array}{ll}
23 & 23 \\
25 & 25
\end{array}
\end{aligned}
$$

from which the increase of $x$ with the size of the particles is put beyond question and the larger coefficient of survival for vapor nuclei as compared with ions is again apparent. Whether the peculiar features of the curve, Fig. $5 c$, which reappears in each case, have a definite meaning must be left to conjecture ; but in most of the curves, $a, b, c, d$, the occurrence of maximum $x$ is in evidence.

6. Loss of Nuclei Actually Due to Evraporation. - It is finally to be shown that the peculiar loss of water nuclei resulting after evaporation of fog particles precipitated upon ions is due to this evaporation (or its equivalent) and not to the dissipation of the water nuclei in the lapse of time. It might be supposed for instance that water nuclei obtained from the fog condensed on the ions are smaller and therefore diffuse more rapidly than water nuclei obtained by other methods. If so, then if the time between the successive exhaustions is doubled, trebled, etc., the loss should be correspondingly increased. Data investigated on the above plan but containing the time interval, $t$, in minutes between the exhaustions, shows that the time effect is secondary. The interval $t$ was varied from $I$ to 6 minutes.

If the results are constructed in a figure like the above, the abscissa being the time in minutes, the ordinates showing the nucleation, the curves indicate a steady progression towards the right as the time interval increases, showing that the time losses although not necessarily absent are not of relevant importance. In fact the group of observations for one minute and six minute intervals, constructed in terms of the number of exhaustions (ignoring lapse of time), are virtually coincident. Again the curve for two minute intervals actually shows less loss (due to favorable exhaustion conditions) than the curve for one minute interval. 
In one series of experiments, radium was left in place except during the exhaustion (for ions are efficient in presence of water nuclei). It was found that the water nuclei stored in this ionized field do not decay more rapidly than in ordinary dust free wet air.

BROWN UNIVERSITY, Providence, R. I. 